\title{
NUEVAS PERSPECTIVAS DE LA LASERTERAPIA
}

José H. Amón Sesmero.

Servicio de Urología. Hospital Río Hortega. Valladolid. España.

Resumen.- Hacemos en este trabajo una revisión de las tendencias actuales y de las nuevas perspectivas del uso del láser en urología, analizando los diferentes campos en los que se ha utilizado esta energía.

En litotricia, el láser ha modificando los algoritmos terapéuticos de la litiasis ureteral. Además, su posibilidad de empleo en ureteroscopia flexible está permitiendo realizar la litotricia intracorpórea "in situ" a nivel renal. Nuevos láseres como el Erbium: YAG, más eficaces y más inocuos que el holmium están actualmente en desarrollo. La investigación en nuevas fibras, más flexibles, económicas y duraderas es el próximo reto en este campo.

En HBP, si bien el láser KTP ha conseguido una difusión extraordinaria para un determinado tipo de volumen prostático, nuevos láseres como el Thulium y el Diodo, dada la precisión de su corte, compiten con el Holmium en el terreno enucleación prostática.

La aplicación del láser en la terapia fotodinámica del cáncer urológico y en la soldadura de tejidos constituyen en la actualidad nuevos objetivos de investigación prometedores.

Palabras clave: Nuevas tendencias. Thulium. Erbium. Terapia fotodinámica. Soldadura de tejidos. Láser.

Summary.- We review the current trends and new perspectives of the use of laser in urology, analyzing different fields in which this energy has been used.

In lithotripsy, the laser has modified the therapeutic algorithms for ureteral lithiasis. Moreover, the possibility of using laser with flexible ureteroscopes is enabling the performance of intracorporeal in situ renal lithotripsy. New lasers such as Erbium:yag, more effective and more innocuous than holmium are currently under development. The research in new fibers, more flexible, economic and long-lasting is the next challenge in this field.

In BPH, although the KTP laser has obtained and extraordinary diffusion for a certain type of prostate volume, new lasers such as thulium and diode, are in competition with holmium laser in the field of prostatic enucleation due to their precise cut.

The application of laser in photodynamic therapy for urological cancers and tissue welding are currently new promising objectives of research.

Keywords: New trends. Thulium. Erbium. Photodinamic therapy. Tissue welding. Laser.
José H. Amón Sesmero

C/ Colonia, 14 - 2

Pinar de Antequera.

47153 Valladolid. (España).

amonsesmero@yahoo.es 


\section{INTRODUCCIÓN}

La energía láser ha tenido amplia expansión en diferentes campos de la medicina como la oftalmología, dermatología y urología. Concretamente, en ésta última su utilización es antigua. Fue Parson en 1966 el primero en utilizar la radiación láser de forma experimental en la vejigas caninas, y Mulvany, en 1968, el primero en conseguir la litofragmentación de un cálculo con un láser de rubí que, finalmente, fue abandonado debido al daño tisular que producía (1).

El desarrollo del láser prosiguió y durante los últimos veinte años se ha utilizado en diferentes patologías urológicas: HBP, litiasis, cáncer de urotelio, estenosis de la vía excretora y patología tumoral de genitales.

Antiguos láseres como el argón y el dye han sido reemplazados por los actuales, de estado sólido (Nd:YAG, KTP, Ho: YAG), que resultan más efectivos, compactos, potentes y económicos. Pero además los avances en la tecnología han conseguido fibras más pequeñas y flexibles que pueden ser utilizadas con los modernos ureterorrenoscopios flexibles, lo que ha reverdecido el interés del uso del láser en cirugía minimamente invasora.

En este trabajo revisamos las tendencias más recientes en laserterapia en urología junto a los nuevos láseres en desarrollo.

\section{Cómo trabajan los láseres en patología urológica}

Para entender las limitaciones de los láseres actuales y las aportaciones de los nuevos es necesario recordar como se produce la interacción láser-tejido en base a los principios físicos de este tipo de radiación.

La mayoría de los láseres de aplicación médica actúan por combinación en mayor o menor medida de tres efectos: fototérmico, fotomecánico y fotoquímico. La consecuencia del efecto fototérmico en el tejido que absorbe la radiación es la elevación de la temperatura produciendo incisión y ablación. La constitución química de los diferentes componentes de un tejido es la que determina la específica longitud de onda de radiación electromagnética que es absorbida eficazmente por él, lo que se conoce como "pico de absorción". El agua y la hemoglobina son los dos componentes comunes de los tejidos corporales con dos picos de absorción de 1,910 y $2870 \mathrm{~nm}$ para la primera, y uno de $532 \mathrm{~nm}$ para la segunda. El efecto fototérmico se facilita cuanto mas cerca esté la longitud de onda del láser utilizado del pico de absorción de las sustancias que componen dicho tejido. Este es el mecanismo de acción de los láseres utilizados en HBP, patología tumoral y estenosis.

El efecto fotomecánico o fotoacústico lo producen los láseres que emiten la energía de forma discontinua en series de pulsos en vez de hacerlo en forma de onda continua. Esta forma de emisión de energía produce una evaporación instantánea del fluido creándose por cavitación una burbuja de plasma que se expande hasta un tamaño máximo para colapsarse bruscamente, lo que genera una fuerte onda de choque en la punta de la fibra láser que se transmite a través del fluido hacia la zona a tratar. Este es el principio con el trabajan los láseres utilizados en fragmentación litiásica.

También la energía láser puede desencadenar una reacción química en el tejido que modifique sus características físicoquímicas. El efecto fotoquímico ha sido hasta ahora el que menos aplicaciones ha encontrado en urología, si bien los avances en terapia fotodinámica de los tumores harán que se usen en un próximo futuro.

Uno de los retos de la energía láser aún sin resolver, es su aplicación precisa sin efectos colaterales en los tejidos circundantes a la lesión. Como explicábamos antes, uno de los factores determinantes de la precisión en la aplicación es cómo de próxima es la longitud de onda del láser del pico de absorción del principal componente del tejido a tratar.

\section{Tendencias actuales y nuevas perspectivas en litotricia}

A mediados de la década de los 80 se generalizó el uso del láser pulsado colorante. Candela introduce en 1986 un voluminoso láser que trabaja con un pulso corto de $1 \mu$ s y una longitud de onda de $504 \mathrm{~nm}$, que consigue la fragmentación litiásica mediante efecto fotoacústico, provocando una onda de choque en la superficie del cálculo al colocar la punta de la fibra de aplicación en contacto con éste. La ausencia de daños colaterales en el urotelio, posibilitaba el poder ser manejado incluso en condiciones de baja o nula visibilidad endoscópica, lo que representaba una gran ventaja. Su eficacia en el tratamiento de todo tipo de litiasis, exceptuando los cálculos de cistina y brushita, ha sido ampliamente demostrada (2), pero su gran tamaño, elevado coste y mantenimiento hizo disminuir su empleo a favor de otros equipos más compactos y económicos como son el láser FREDDY y el Ho: YAG.

El láser FREDDY (acrónimo de frequency-doubled double pulse laser Neodyn: YAG) aprobado por la FDA americana en 2001, constituye una interesante innovación en litotricia, dado su bajo coste y reducido tamaño. Este láser trabaja con un doble pulso de corta duración(1 $1 \mu s)$, combinando dos longitudes de onda (532 y $1064 \mathrm{~nm}$ ) mediante la incorporación de un cristal de KTP al resonador de un láser Nd: YAG. La luz de $532 \mathrm{~nm}$ de longitud de onda es absorbida por el cálculo iniciando la formación de plasma en su superficie, y la luz de 1064 nm calienta el plasma preformado, lo que provoca expansión y posterior contracción de la burbuja de plasma. Este mecanismo fotoacústico tiene la ventaja de no producir daño colateral, como ha podido ser demostrado en estudios experimentales, por lo que puede ser utilizado en condiciones de escasa visibilidad en la endoscopia (3). Además, es más económico que otros láseres.

Pero a pesar de estas notables ventajas, su utilización tiene carencias a tener en cuenta, ya que se ha comunicado falta de efectividad en los cálculos duros, donde también fracasaba el láser colorante: cálculos de cistina, brushita y oxalato cálcico monohidrato $(4,5)$. 
Actualmente, el Holmium YAG (YTTRIUM-LumenGarnet) es el láser más utilizado en litotricia dado su menor tamaño, coste y mantenimiento. Aunque es manufacturado por diferentes fabricantes, la mayoría de ellos utilizan una longitud de onda de $2120 \mathrm{~nm}$ y un pulso que varía de 350 a 500 ps. Esta longitud de onda está próxima a la de absorción del agua (1940), lo que proporciona seguridad en el medio acuoso que utiliza la endoscopia (6).

A medida que la energía óptica es absorbida por el cálculo, la temperatura en su superficie aumenta hasta un nivel crítico que produce pequeños cráteres en su superficie por descomposición química de los componentes de la litiasis. Este mecanismo de acción provoca una litotricia en pequeños fragmentos produciendo el efecto endoscópico de tallado o "resección" del calculo como algunos autores han señalado $(7,8)$. La pequeña duración del pulso minimiza el fenómeno fotoacústico, predominando el efecto fototérmico como mecanismo de fragmentación. Esto determina que disminuya notablemente el efecto negativo de la retropulsión del cálculo que se observa en los láseres que utilizan un mecanismo fotoacústico, si bien es un proceso más lento que otros $(7,8)$.

El láser Ho: YAG ha demostrado una alta eficacia en la resolución clínica de litiasis de cualquier composición química produciendo menores fragmentos que otros láseres, disminuyendo así el número de maniobras auxiliares. Su seguridad en cuanto al daño que puede producir en la pared ureteral es alta siempre que la punta de la fibra se sitúe a una distancia mayor de $0,5 \mathrm{~cm}$., en la que el agua intermedia absorba la energía $(7,9)$.

El Holmium utiliza fibras de silicato de cuarzo de 200 y 365 micrones muy flexibles lo que permite su paso por ureteroscopios flexibles 7,5 Fr. La fibra de 200 micrones permite ser usada con la máxima deflexión y buena irrigación pero corre el riesgo de rotura en la punta cuando el nivel de energía supera 1 julio (10).

\section{Nuevas tendencias en litotricia}

En la actualidad se esta desarrollando el láser Erbium: YAG, que a la vista de los estudios experimentales realizados puede ser un eficaz competidor del Holmium: YAG en el campo de la fragmentación litiásica (11).

Estudios experimentales demuestran que los cálculos absorben la máxima energía para una longitud de onda de $2940 \mathrm{~nm}$, lo que corresponde a la longitud de onda en coincidencia con el pico de absorción del agua en el espectro infrarrojo (12).

La corta duración del pulso con el que trabaja este láser, minimiza el efecto de retropulsión del cálculo. Teichman demostró con experimentos" in vitro" que aunque el láser Erbium fragmenta las litiasis mediante un efecto fototérmico, al igual que el Holmium, era de tres a cinco veces más eficiente que este último en la fragmentación de las litiasis duras como las de cistina y oxalato cálcico monohidrato (8). Esta superior capacidad de trabajo es debida a la particular forma de torpedo que adquiere la burbuja de plasma que crea, produciendo un cráter más liso y profundo el la superficie de la litiasis que el Ho: YAG (12).

Así pues, hay menor irradiación de energía(menos daño colateral) y mayor eficacia con la longitud de onda del Erbium: YAG que con la del Holmium: YAG.

Pero un inconveniente que ha frenado el desarrollo de este láser ha sido hasta ahora la falta de fibras adecuadas para su aplicación. Las fibras de silicato de cuarzo, que tan bien funcionan con el Holmium, absorben la energía óptica de este láser y sufren una degradación térmica, que incluso puede dañar el ureterorrenoscopio. Estudios con fibras de zafiro han demostrado que si bien trasmiten la energía con escasas pérdidas, debido al elevado pico de densidad de energía que se produce en su punta, ésta se deteriora. Este hecho y su alto precio son hoy por hoy inconvenientes para la generalización del empleo de este láser (13).

La utilización del láser en litotricia está modificando los algoritmos terapéuticos de la litiasis. La mayoría de las series de ureteroscopia con láser Holmium alcanzan una tasa de éxito de resolución de la litiasis superior al 95\%, incluso cuando esta asienta en el uréter próximal( 14,15$)$. Y como explicábamos arriba, una de las ventajas del láser es su posibilidad de empleo en ureteroscopia flexible para acceder a la litiasis del uréter próximal o incluso a la litiasis intrarrenal.

Hasta ahora la litiasis renal de gran tamaño o compleja era tributaria de cirugía percutánea renal, lo que implicaba una proporción no desdeñable de abordajes supracostales o múltiples accesos, aumentando el riesgo de complicaciones torácicas y sangrado $(16,17)$. Los recientes avances en ureteroscopia flexible e instrumentación de nitinol, así como el uso de vainas de acceso ureteral, han permitido realizar la litotricia intracorpórea "in situ" a bajas presiones $(18,19,20)$. La combinación de ambas técnicas, NLP y ureteroscopia flexible en las litiasis de gran tamaño o complejas, evitando la realización de varios tractos se ha mostrado muy eficaz $(21,22)$.

\section{Tendencias actuales y nuevas perspectivas en hiperplasia benigna de próstata}

El tratamiento de la hiperplasia benigna de próstata mediante energía láser ha evolucionado de la coagulación a la enucleación. Los sistemas ILC (coagulación intersticial con láser), y VLAP (ablación visual con láser de la próstata), producían la ablación del tejido prostático mediante una necrosis de coagulación. El láser utilizado en la ILC era el Nd: YAG con una longitud de onda de 1064 $\mathrm{nm}$. Este láser, cuya radiación era invisible, se aplicaba en modalidad de contacto. Su eficacia se demostró nula, pues si bien su bajo coeficiente de absorción por la mayoría de los tejidos le proporcionaba una profunda penetración, la baja densidad de energía producía en los tejidos un calentamiento inferior al punto de ebullición que sólo conseguía una necrosis de coagulación. La lesión intraprostática sufría reabsorción y atrofia secundaria por un proceso de 
retracción a lo largo de varias semanas. Los estudios comparativos con la RTU pusieron en evidencia menor mejoría del flujo y de la escala de síntomas y mayor tasa de reintervención para esta técnica que para la RTU $(23,24)$.

EI VLAP utilizaba el Nd: YAG a través de una fibra que deflectaba la punta unos $90^{\circ}$ para dirigir la luz láser sin contacto con el tejido, creando una profunda necrosis de coagulación. La eliminación del tejido necrótico por la uretra creaba una cavidad que posteriormente se epitelizaba. También con este láser, los estudios comparativos randomizados que lo compararon con la RTU demostraron menor reducción de volumen, menor reducción en la escala de síntomas, menor tasa de flujo urinario y mayor residuo para el VLAP que para aquélla $(25,26)$. El metaanálisis puso en evidencia que la tasa de reintervención con VLAP varió de 7,5-20\% durante el primer año (26) y alcanzó el $26,7 \%$ en el segundo año de seguimiento (25). Esta alta tasa de reintervención y la necesidad de cateterismo prolongado y postergaron el empleo de estos láseres.

Posteriormente se ha desarrollado un Nd: YAG de doble frecuencia, lo que se consigue haciendo pasar el rayo de $1064 \mathrm{~nm}$ de longitud de onda a través de un cristal de potasio-titanio-fosfato que lo trasforma en un rayo de mitad longitud de onda (532 nm) en el espectro de la luz verde y de doble frecuencia (11). Este color de la luz o el acrónimo de los componentes del cristal es como se conoce a este láser: KTP o láser de "luz verde".

El KTP es fuertemente absorbido por la hemoglobina, lo que consigue una menos profunda penetración y más efectiva ablación de tejido con menor zona de coagulación. Esta ablación conocida como vaporización fotoselectiva de la próstata fue iniciada por Malek (27), ganó popularidad con los láseres KTP de de 80 w $(28,29,30)$. La inmediata vaporización del tejido prostático obstructivo permite una recuperación de la micción rápida, reduciendo el cateterismo uretral sensiblemente respecto a la RTU (31). Su mayor inconveniente, la necesidad de excesivo tiempo para conseguir la vaporización del estroma prostático, ha sido contrarestado con un progresivo aumento de potencia a a $120 \mathrm{~W}$, consiguiéndose estandarizar la técnica en tiempos aceptables al compararlos con los de la RTU (32). Pero es indudable que se necesita asegurar la estabilidad de los resultados con mayor tiempo de seguimiento.

El Ho: YAG no solo se utiliza para litofragmentación sino también para la enucleación de la próstata. Su longitud de onda de $2140 \mathrm{~nm}$ es fuertemente absorbida por el agua con una penetración de solo 0,4 mm. La escasa penetración y la alta fluencia de este láser hace que la densidad de energía sea lo suficientemente alta para calentar el tejido por encima de los $100^{\circ} \mathrm{C}$ produciéndose predominantemente vaporización con escasa coagulación profunda(33). Este mecanismo de acción, a diferencia del $\mathrm{Nd}$ : YAG que por su mayor penetración y menor fluencia produce lesión térmica y coagulación, causa mínimas lesiones en el lecho prostático reduciendo significativamente el tiempo de sonda uretral (34).

Este láser ha sido utilizado para vaporizar la próstata (HoLAP) con fibras de haz lateral que se manejan como el láser KTP, y para enuclear la próstata (HoLRP/ HoLEP).

La vaporización crea una cavidad exangüe que permite una precoz retirada de la sonda uretral y tiene una corta curva de aprendizaje. Dado que la vaporización es un proceso muy lento, en el que solo se destruye 0,5 gramos de tejido al minuto, parece solo adecuada para pequeños adenomas (35). En un estudio prospectivo randomizado que la compara con la RTUP en pequeños adenomas, se observa igual efectividad al año de seguimiento (36).

La enucleación prostática con HoLRP y HoLEP ha sido comparada con la RTUP en varios estudios prospectivos randomizados (36-39) demostrándose mejoría de la nicturia de los pacientes tratados. Además, HoLEP es la única alternativa que ha demostrado ser superior a la RTUP al valorar los parámetros urodinámicos del vaciamiento vesical $(40,41)$.

Una interesante innovación lo constituye el láser Thulium:YAG (RevoLix). Este láser infrarrojo recientemente comercializado, está basado en el desarrollo tecnológico de las fibras. Trabaja con una longitud de onda de 2,0 micrones, cercana al pico de absorción del agua por lo que puede ser utilizado para la ablación de la próstata y, además, ser empleado en litotricia.

Este láser utiliza la propia fibra de thulium como medio de amplificación, siendo estimulada por un láser diodo, lo que permite modificar la forma de trabajo de modo pulsado a continuo. Esto supone una ventaja frente a el Ho:YAG, ya que cuando se utiliza de modo no pulsado (onda continua), no se producen las burbujas de vapor de agua que aparecen con el Holmium, lo que permite realizar suaves y precisas incisiones. Además, la posibilidad de ajustar la longitud de onda de acuerdo a la profundidad que queramos obtener en el tejido permite incrementar la seguridad. Los estudios experimentales en animal de experimentación utilizando este láser para la ablación de tejido parecen ser prometedores (42). Fried ha llevado a cabo la ablación de próstatas caninas mediante vaporización con láser Thulium, consiguiendo una zona de coagulación de $0,05-0,2 \mathrm{~mm}$, que permite realizar una buena hemostasia (44).

También, recientes estudios clínicos han demostrado que la resección de la próstata con láser Thulium se realiza con mínimo sangrado y hospitalización, y ausencia de complicaciones (43). Los parámetros de flujo postoperatorio y puntuación sintomática mostraron franca mejoría, por lo que se podría considerar eficaz este láser en la ablación prostática, si bien se precisan más estudios por ahora.

El láser Diodo utiliza luz eléctrica que es directamente transformada en radiación láser a diferencia de los de $\mathrm{Nd}$ : YAG y Ho: YAG en los que el cristal láser necesita ser estimulado directamente por la luz generada por una lámpara o un láser diodo. Esto posibilita ventajas como que el tamaño de su diseño y su coste sean reducidos, si bien su mayor problema es su limitación para focalizar suficiente nivel de potencia en fibras de pequeño calibre. 
El láser diodo $\mathrm{Nd}$ : YAG ha sido empleado en para conseguir una amplia y profunda coagulación por calor de la próstata sin vaporización, lo que se conoce como coagulación intersticial por láser (ILC). Existen actualmente láseres diodo de 890,980 y $1460 \mathrm{~nm}$ de longitud de onda que actúan con penetraciones que varían de milímetros a centímetros en el tejido prostático. La longitud de onda de $980 \mathrm{~nm}$ ofrece una simultánea absorción por el agua y la hemoglobina, lo que permite una alta resolución ablativa con buena hemostasia a la vista de estudios experimentales comparativos con el láser KTP y la RTUP en riñones porcinos (45), y de los primeros estudios clínicos descriptivos (46).

\section{Nuevas tendencias del láser en oncología urológica}

Una interesante aplicación de la energía láser es la terapia fotodinámica de los tumores. El mecanismo de acción de este tratamiento es la reacción química que genera la luz electromagnética en las células tumorales, previamente sensibilizadas mediante la administración de una sustancia fotosensible, que causa una destrucción celular selectiva de células neoplásicas (11). La reacción química intracelular que se genera, del tipo radical libre de oxígeno, $q$ es nociva para las células. Especialmente sensibles son las del endotelio de las vasos por lo que la radiación provocará una destrucción de microcirculación y, por tanto, del tumor.

Aquellos órganos en los que se puede administrar altas dosis de fotosensibilizador sobre la lesión gracias a su accesibilidad son potencialmente idóneos para este tratamiento como es el caso de los tumores vesicales, accesibles por endoscopia, y los tumores epiteliales del pene, en los que se está investigando con ácido aminolevulínico y hematoporfirina como fotosensibilizadores $(47,48)$.

También se ha realizado este tipo de terapia por vía intersticial para el tratamiento de tumores de órganos sólidos, especialmente en cáncer de próstata, aunque no hay todavía resultados publicados al respecto. Conocer el grado de aplicación de la energía láser que conseguirá desencadenar la reacción química es el desafío que tienen estas terapias así como encontrar sustancias fotosensibilizantes con menos efectos colaterales(49).

Además, la reacción química mediada por fotosensibilización celular puede ser usada, no para destruir la célula tumoral, sino para su identificación y posterior tratamiento con otras alternativas. Especialmente en el seguimiento de los tumores vesicales superficiales recidivantes, en los que la cistoscopia tiene limitaciones en identificar lesiones iniciales, el diagnóstico espectroscópico puede ser muy útil (50).

\section{Nuevas aplicaciones de láser en estenosis}

El láser Er: YAG, además de ser más eficiente litotriptor que el Ho:YAG, tiene otra ventaja: la profundidad de penetración en tejidos blandos $(30 \mu \mathrm{m})$ es mucho menor que la del Holmium (200-400 $\mu \mathrm{m})$, lo que disminuye el grado de lesión en el tejido circundante. La precisión con la que realiza la incisión es especialmente útil para el tratamiento de estenosis y no en cambio para la ablación de grandes cantidades de tejido, dada su lentitud de trabajo. Por otro lado, al no producir una zona de coagulación amplia su poder de hemostasia es muy limitado (11).

También el láser de fibra de Thulium ofrece posibilidades para la incisión de estenosis cuando es usado en forma pulsada. Dado que trabaja con una longitud de onda inferior a $2400 \mathrm{~nm}$, puede utilizar las fibras de sílice regulares con alta transmisión de energía y posibilidad de ser utilizadas en endoscopia flexibles, lo que está vedado para el láser Erbium. Pero la profundidad de su incisión, similar a la del Holmium, hace que su precisión sea inferior a la del Erbium en esta tipo de tratamientos.

\section{Aplicaciones del láser en la soldadura de tejidos}

La energía láser ha sido utilizada para la reaproximación de tejidos en especialidades como la oftalmología y se ha utilizado experimentalmente en urología para el sellado de la ureterotomía en modelo porcino (51). El láser "soldador" puede actuar de una forma simple, calentando los tejidos y poniéndolos en contacto sin el empleo de sustancias que faciliten la adhesión. Se ha utilizado el láser Diodo en la soldadura de tejidos como la piel, una vez enfrentados sus bordes, mediante la producción de calor en los mismos (52).

Otra forma de utilización consiste en emplear un adhesivo biológico que al contener un cromóforo que absorbe la luz láser, hace de "soldador" facilitando la aproximación del tejido y el sellado de órganos huecos, evitando así fugas de fluidos. Se ha utilizado fibrinogeno como agente soldador, con indocianida verde como cromóforo (53). También, el láser puede actuar estimulando fototérmicamente una sustancia capaz de crear enlaces covalentes con el tejido que se quiere cicatrizar. Recientemente se ha comunicado el desarrollo de un sistema láser con control de la temperatura capaz de unir incisiones en la piel porcina usando suero de albúmina semisólida mediante un dispositivo da aproximación. El procedimiento es más rápido y no-dependiente del cirujano, aísla la herida del entorno inmediatamente, disminuyendo la tasa de infección y mejorando los resultados cosméticos. Si bien estos estudios experimentales han sido realizados en piel porcina, la similitud de ésta con la humana hace que estos resultados sean prometedores. La aplicabilidad de este sistema por vía laparoscópica (54) en la reparación de la unión pieloureteral del modelo porcino abre un campo novedoso en urología.

\section{CONCLUSIONES}

Existen nuevos láseres con aplicaciones en urología que están siendo objeto de estudio. En el campo de la litotricia, su empleo futuro va a incrementarse notablemente en el terreno de la ureteroscopia flexible para el tratamiento de las litiasis del uréter próximal e intrarrenal. Si bien hay interesantes expectativas en el empleo del láser 
Erbium, dada su mayor eficacia frente al Holmium, se precisa investigar en el desarrollo de fibras adecuadas para su uso en ureteroscopia flexible.

También el campo de la HBP se está experimentando con nuevos láseres y la tendencia en el futuro próximo va a ser el empleo de esta energía en la resolución de la hiperplasia de próstata obstructiva. El láser KTP tendrá unas aplicaciones concretas en esta patología dada su eficacia en la vaporización y coagulación. El láser Erbium: YAG y el láser de $\mathrm{CO} 2$ consiguen una incisión más precisa en la enucleación prostática que el láser Holmium, por lo que podrían ser una franca alternativa si se consiguen superar los inconvenientes de sus actuales fibras. El láser Thulium no tiene ese problema, y podría sustituir al Holmium en muchas aplicaciones, pero actualmente su aplicación es cara debido al uso de un láser diodo como fuente.

La terapia fotodinámica de los tumores abre un campo especialmente sugerente en el cáncer de próstata, aunque se precisa mayor investigación en la aplicación láser y en fármacos sensibilizantes.

Finalmente, los láseres para soldadura de tejidos tienen un especial interés en patología urológica, dada la necesidad de conseguir la estanqueidad de la vía urinarias cuando intervenimos en ella. Su desarrollo, aunque todavía experimental, parece prometedor.

\section{BIBLIOGRAFÍA Y LECTURAS RECOMENDADAS ("lectura de interés $y$ ** lectura fundamental)}

1. GROSS, A.J.; HERRMAN, T.R.: "History of lasers". World J. Urol., 25: 227, 2007.

2. STEIN, B.S.; KENDALL, A.R.: "Lasers in urology II. Laser therapy". Urology, 23: 411, 1984.

3. SANTA CRUZ, R.W.; LEVEILLEE, R.J.; KRONGRAD, A. y cols.: "Ex vivo comparison of tour lithotripters commonly used in ureter : What does it take to perforate?". J. Endourol., 12: 147, 1998.

4. DUBOSQ, F.; PASQUI, F.; GIRARD, F. y cols.: "Endoscopic lithotripsy and FREDDEY laser: Inicial experience". J. Endourol., 20: 296, 2006.

5. STARK, L.; CAR, P.: "First clinical experiences of laser lithotripsy using the partially frequency-doubled double pulse neodimyum: YAG laser (FREDDY)". J. Urol., 165: 362, 2001.

6. CHAN, K.F.; VASSAR, G.J.; PFEFER, T.J. y cols.: "Holmium: YAG laser lithotripsy: A dominant photothermal ablative mechanism with chemical decomposition of urinary calculi". Laser Surg. Med., 25: 22, 1999.

7. GRASSO, M.: "Experience with the holmium laser as an endoscopic lithotrite". Urology, 48: 199, 1996.

8. TEICHMAN, J.M.; VASSAR, G.J.; BISHOFF, J.T. y cols.: "Holmium: YAG lithotripsy yields smaller fragments than lithoclats, pulsed dye laser or electrohydraulic lithotripsy”. J. Urol., 159: 17, 1998.
9. RAZVI, H.A.; DENSTEDT, J.D.; CHUN, S.S. y cols.: "Intracorporeal lithotrypsy with the Holmium: YAG laser". J. Urol., 156: 912, 1996.

10. MONGA, M.; BEST, S.; VENKATESH, R. y cols.: "Durability of flexible ureteroscopes: A randomized, prospective study". J. Urol., 176: 137, 2006.

*11. FRIED, M.: "Therapeutic applications of lasers in urology: An update". Expert. Rev. Med. Devices., 3: 31, 2006.

12. CHAN, K.F.; HAMMER, D.X.; CHOI, B. y cols.: "Free electron laser lithotripsy: Threshold radiant exposures". J. Endourol., 14: 161, 2000.

13. TEICHMAN, J.M.; CHAN, K.F.; CECCONI, P.P. y cols: "Erbium: YAG versus Holmium: YAG". J. Urol., 165: 876, 2001.

14. SOFER, M.; WATTERSON, J.D.; WOLIN, T.A. y cols.: "Holmium: YAG lithotripsy for upper tract urinary calculi in 598 patients". J. Urol., 167: 31, 2002.

15. LAM, S.M.; WATTERSON, J.D.; WOLIN, T.A. y cols.: "Holmium: YAG lithotripsy for upper tract urinary calculi in 598 patients". J. Urol., 167: 31, 2002.

16. LAM, J.S.; GREEN, T.D.; GUPTA, M. y cols.: "Treatment of proximal ureteral calculi: Holmium: YAG laser ureterolithotripsy versus extracorporeal shock wave lithotripsy". J. Urol., 167: 1972, 2002.

17. MUNVER, R.; DELVECCHIO, F.C.; NEWMAN, G.M. y cols.: "Citical análisis of supracostal access for percutaneous renal surgery". J. Urol., 166: 1242, 2001.

18. EL-ANANY, F.G.; HAMMOUDA, H.M.; MAGHARABY, H.A. y cols.: "Retrograde ureteropyeloscopic holmium laser lithotripsy for large renal calculi". BJU. Int., 88: 850, 2001.

19. KOURAMBAS, J.; BYRNE, R.R.; PREMINGER, G.M.: "Does a ureteral access sheath facilitate ureteroscopy?". J. Urol., 165: 789, 2001.

20. AUGE, B.K.; PIETROW, P.K.; LALLAS, C.D. y cols.: "Ureteral access sheath provides protection against elevated renal pressures during rutine flexible ureteroscopic manipulation”. J. Endourol., 18: 33, 2004.

21. LANDMAN, J.; VENKATESH, R.; LEE, D.I. y cols.: "Combined percutaneous and retrograde approach to staghorn calculi with application of the ureteral access sheath to facilitate percutaneous nephrolithotomy". J. Urol., 169: 64, 2003.

22. MARGUET, C.G.; SPRINGHART, W.T.; TAN,Y.H. y cols.: "Simultaneous combined use of flexibleureteroscopy and percutaneous nephrolithotomy to reduce the number of access tracts in the management of complex renal calculi”. BJU. Int., 96: 1097, 2005.

23. MUSCHTER.; WHITFIELD.: "Intersticial laser therapy of benign prostatic hyperplasia". Eur. Urol., 35: 147, 1999.

24. LAGUNA, G.; ALIVIZATOS, G.; DE LA ROSETTE.: "Intersticial laser coagulation treatment of benign prostatic hyperplasia: Is it to be recommended?". J. Endourol., 17: 595, 2003.

25. WHEELAHAN, J.; SCOTT, N.A.; CARTMILL, R. y cols.: "Minimally invasive laser techniques for prostatectomy: A systematic rewiew". BJU. Int., 86: 805, 2000.

*26. HOFFMAN, R.M.; MACDONALD, R.; SLATON, J.W. y cols.: "Laser prostatectomy versus transurethral resection for treating benign prostatic obstruction: A 
systematic rewiew". J. Urol., 169: 210, 2003.

27. MALEK, R.S.; KUNTZMAN.; BARRETT.: "Photoselective potassium-titanyl- phosphate laser vaporization of the benign obstructive prostate: Observation on long-term outcomes". J. Urol., 174: 1344, 2005.

28. SULSER, T.; REICH, O.; WYLER, S. y cols.: "Photoselective KTP laser vaporization of the prostate: First experiences with 65 procedures". J. Endourol., 18: 976, 2004.

29. SANDHU, J.S.; NG, C.; VANDERBRINK, C. y cols.: "High power potassium-titanyl-phosphate photoselective laser vaporization of prostate for treatment of benign prostates hyperplasia in men with large prostates". Urology, 64: 1155, 2004.

30. GOMEZ SANCHA, F.; CASTILLON-VELA, I.: "KTP laser photoselective vaporization of the prostate: Single surgeon experience on 150 patients". J. Urol., 175: 491, 2006.

31. VOLKAN, T.; IHSAN, T.A.; YILMAZ, O. y cols.: "Short-term outcomes of high power $(80 \mathrm{~W})$ potassium-titanyl-phosphate laser vaporization of the prostate". Eur. Urol., 48: 608, 2005.

*32. GOMEZ SANCHA, F.; BACHMANN, A.; CHOI, B.B. y cols.: "Photoselective vaporization of the prostate: Lessons learnt after 3500 procedures". Prostate Cancer Prostatic. Dis., 10: 316, 2007.

33. JENSEN, E.D.; VAN LEEUWEN, T.G.; MOTAMEDI, M. y cols.: "Temperatura dependency of the absorption coefficient of water for mid-infrared laser radiation". Laser Surg. Med., 14: 258, 1994.

*34. KUNTZ, R.M.: "Current role of lasers in the treatment of benign prostatic hyperplasia (BPH)". Eur. Urology., 49: 961, 2006.

35. TE, A.E.; MALLOY, T.R.; STEIN, B.S. y cols.: "Photoselective vaporization of the prostate for the treatment of benign prostatic hyperplasia: 12 month reslts from the first United States multicenter prospective trial". J. Urol., 172: 1404, 2004.

36. MOTTET, M.; ANIDJAR, M.; BOURDON, O. y cols.: "Randomized comparison of transurethral electroresection and holmium: YAG laser vaporization for symptomatic benign prostatic hyperplasia". J. Endourol., 13: 127, 1999.

37. TAN. P.J.; GILLING.; KENNETT, C. y cols.: "A rndomized trial comparing Holmium laser enucleation of the prostate with transurethral resection of the prostate for the treatment of bladder outlet obstruction secondary to benign prostatic hyperplasia in large glands (40 to 200 grams)". J. Urol., 170: 1270, 2003.

38. MONTORSI, F.; NASPRO, R.; SALONIA, A. y cols.: "Holmium laser enucleation versus transurethral resection of the prostate: Results from a 2 center, prospective, randomized trial in patients with obstructive benign prostatic hyperplasia”. J. Urol., 172: 1926, 2004.

39. KUNTZ, R.M.; AHYAI, S.; LEHRICH, K. y cols.: "Transurethral Holmium laser enucleation of the prostate versus transurethral electrocautery resection of the prostate: A randomized prospective trial in 200 patients". J. Urol., 172: 1012, 2004.

40. GILLING, P.J.; MACKEY, M.; CRESSWELL, M. y cols.: "Holmium laser versus transurethral resection of the prostate: A randomized prospective trial with 1 year follow up". J. Urol., 162: 1640, 1999.

41. LIAM, C.W.; GILLING, P.J.; WILLIAMS, A. y cols.: "A randomized trial comparing Holmium laser enucleation versus transurethral resection in the treatment of prostates larger than 40 grams: Results at 2 years". Eur. Urol., 50: 569, 2006.

42. HEISEN, D.; OTT, V.; BERN, H. y cols.: "Cw high power IR-laser at 2 microns for minimally invasive surgery". Proceedings of SPIE, 5142: 96, 2003.

**43. MARKS, A.J.; TEICHMAN, J.M.: "Lasers in clinical urology: State of the art and new horizons".

44. FRIED, N.M.; MURRAY, K.E.: "High power thulium fiber laser ablation of urinary tissues at 1,94 microm". J. Endourol., 19: 25, 2005.

45. WENDT-NORDAHL, G.; HUCKELE, S.; HONECK, P. y cols: "980-nm Diode laser: A novel laser technology for vaporization of the prostate". Eur. Urol., 52: 1723, 2007.

46. SEITZ, M.; SROKA, R.; GRATZKE, C. y cols.: "The diode laser: A novel side-firing approach for laser vaporisation of the human prostate immediate efficacy and 1 year follow up". Eur. Urol., 52: 1717, 2007.

47. PINTHUS, J.H.; KLEINMAM, N.; TISDALE, B. y cols.: "Lower plasma adiponectin levels are associated with larger tumor size and metastasis in clear-cell carcinoma of the kidney". J. Urol., 175: 1201, 2006.

48. WAIDELICH, R.; BEYER, W.; KNÜCHEL, R. y cols.: "Whole bladder photodynamic therapy with 5-aminolevulinic acid using a white light source". Urology, 61: 332, 2003.

**49. PIERRE, S.A.; ALBALA, D.M.: "The future of lasers in urology". World J. Urol., 25: 275, 2007.

50. OLIVO, M.; LAU, W.; MANIVAGASER, V. y cols.: "Novel photodynamic diagnosis of bladder cancer: Ex vivo fluorescence cytology using hypericin". Int. J. Oncol., 23: 1501, 2003.

51. WOLF, J.S.; SOBLE, J.J.; RAYALA, H.J. y cols.: "Comparison of fibrin glue, laser weld and mechanical suturing device for the laparoscopic closure of ureterotomy in a porcine model”. J. Urol., 157: 1487, 1997.

52. GULSOY, M.; DERELI, Z.; TABAKOGLU, H.O. y cols.: "Closure of skin incisions by $980-\mathrm{nm}$ diode laser welding". Lasers Med. Sci., 21: 5, 2006.

53. SIMHON, D.; HALPERN, M.; BROSH, T. y cols.: "Immediate tight sealing of skin incisions using an innovative temperature-controlled laser soldering device: In vivo study in porcine skin". Ann. Surg., 245: 206, 2007.

54. SHUMALINSKY, D.; LOBICK, L.; CYTRON, S. y cols.: "A laparoscopic laser soldering for repair of ureteropelvic junction obstruction in the porcine model". J. Endourol., 8: 177, 2004. 